\title{
Application value of echocardiography in minimally invasive thoracoscopic mitral valvuloplasty during perioperative period
}

\section{Cui Xiong}

Zhejiang Provincial People's Hospital, Hangzhou Medical College

\section{Yi Zheng}

Zhejiang Provincial People's Hospital, Hangzhou Medical College

\section{Jing Wang}

Zhejiang Provincial People's Hospital, Hangzhou Medical College

\section{Jinghong Xu}

Zhejiang Provincial People's Hospital, Hangzhou Medical College

\section{Qi Hu}

Zhejiang Provincial People's Hospital, Hangzhou Medical College

Ceng Wang ( $\nabla$ 489471921@qq.com )

Zhejiang Provincial People's Hospital, Hangzhou Medical College

\section{Research Article}

Keywords: 3D-Transoesophageal echocardiography (3D-TEE), Transthoracic echocardiography (TTE), perioperative period, mitral regurgitation (MR), mitral valvuloplasty (MVP)

Posted Date: March 29th, 2022

DOI: https://doi.org/10.21203/rs.3.rs-1365133/v2

License: (9) This work is licensed under a Creative Commons Attribution 4.0 International License. Read Full License 


\section{Abstract \\ Objective}

This study retrospectively analysed patients undergoing thoracoscopic mitral valvuloplasty in our hospital from 2019 to 2020 and discussed the value of echocardiography (including three-dimensional transoesophageal echocardiography and transthoracic echocardiography) in the perioperative period.

\section{Methods}

In the Department of Cardiothoracic Surgery of our hospital, 57 patients needing surgical treatment were screened by echocardiography. Ultrasonography was performed preoperatively (G1) and 1 week (G2) and 3 months (G3) postoperatively for each patient. The doctor recorded the left atrial anteroposterior diameter (LAD), left ventricular anteroposterior diameter (LVD), mitral orifice velocity (MV), mitral pressure of gradient (MVG), and ejection fraction (EF), which were used to observe and compare the left heart reconstruction and mitral valve repair to provide an imaging basis for clinicians to choose the most appropriate valve model. Transoesophageal echocardiography was also performed preoperatively to provide an imaging basis for clinicians to choose the most appropriate valve model.

\section{Results}

The results from the three ultrasound examinations showed statistically significant differences among $E F, L A D$, and LVD. The intraoperative transoesophageal ultrasound measurement of the mitral regurgitation area $(S 2,9.57 \pm 1.47)$ was greater than the preoperative transthoracic ultrasound measurement of the mitral regurgitation area ( $\mathrm{S} 1,8.83 \pm 2.01)$, and the difference was statistically significant $(p<0.05)$.

\section{Conclusions}

3D-transoesophageal echocardiography is a convincing imaging examination that can assist cardiologists in formulating the most appropriate surgical plan. Transthoracic echocardiography (TTE) is less invasive and easier to operate compared to transoesophageal echocardiography (TEE). The use of perioperative TTE can help detect new heart conditions in emergency and surgical patients in a timely manner and determine the factors that cause hemodynamic instability, which has a positive effect on the perioperative management of such patients.

\section{Introduction}

The aetiology of mitral regurgitation (MR) is complicated. MR often causes leaflet regurgitation and insufficiency, which can lead to the enlargement of the heart and impaired systolic and diastolic function 
of the heart and affect the quality of life of patients. The prevalence of mitral valve disease has progressed over the past five decades from a disease of primarily rheumatic origin to a disease affecting the aging population, and the disease encompasses a range of phenotypes, such as rheumatic mitral stenosis, degenerative MR, degenerative mitral valve calcification, and secondary MR [1]. MR is a common valvular disorder with an age-dependent prevalence of over $10 \%$ in the elderly population ( $>75$ years) in industrialized countries [2].

The proportion of elderly patients is increasing among patients with mitral valve disease in China. Due to concerns over the risks of surgery, elderly patients with mitral valve disease rarely undergo mitral valve surgery[3]. However, the efficacy of conservative treatments is poor, and surgery remains the best choice. The guidelines of the European Society of Cardiology from 2017 predominantly recommend surgery is obviously indicated in symptomatic patients with severe primary mitral regurgitation [4]. The latest guidelines in 2021 proposed for the first time that non-pharmacological interventions be used for patients with severe secondary MR who still have symptoms after receiving guideline-directed medical therapy. Compared with mitral valve replacement (MVR), mitral valvuloplasty (MVP) has become the first choice for MR treatment due to its long-term effects [5]; MVP can better maintain the structure and morphology of the left ventricle and can reduce the complications related to MVR (such as thrombosis or embolism). Additionally, postoperative anticoagulant treatment is not required with MVP, which reduces complications related to this treatment. Hence, the quality of life of patients is greatly improved. Patients who underwent MVP displayed lower early and long-term mortality, fewer valve-related events and major adverse events [6].

Ultrasound technology provides great value in the preoperative diagnosis, intraoperative monitoring, and postoperative evaluation of mitral valve disease. Transthoracic echocardiography (TTE) can visually display the structure and blood flow of the heart, and it is an important method in evaluating the cardiac function and haemodynamic status during the perioperative period. TTE is convenient, non-invasive, and highly reproducible, making it the first choice for postoperative follow-up. Three-dimensional transoesophageal echocardiography (3D-TEE) technology allows for the real-time display of anatomical perspective images consistent with surgical operations and for quantitative functional analysis. Moreover, 3D-TEE is currently the only imaging examination that can be performed during surgery in real time, making it an indispensable diagnostic and monitoring method for the surgical repair of MR [7]. 3DTEE has become the standard of care for the evaluation of cardiac anatomy and function. Its use offers a new dimension of clinical insight in managing patients for cardiac surgery or structural heart interventions [8].

\section{Materials And Methods}

\section{Patients}

The study was approved by our Institutional Ethics Committee. All patients signed an informed consent form prior to examination. We conducted a retrospective study and selected 58 patients with MR who 
underwent mitral regurgitation repair and long-term postoperative follow-up at Zhejiang Provincial People's Hospital from January 2019 to December 2020. The median age was 54 years and 40 patients (83.3\%) were male. The inclusion criteria were as follows: (1) MR diagnosed with mitral valve insufficiency caused by valve leaflet prolapse, chordae rupture, annulus enlargement, or rheumatic heart disease; (2) no contraindications to transoesophageal echocardiography, such as oesophagus, pharynx, or cervical spine disease. The exclusion criteria were as follows: (1) patients with other complex congenital heart diseases; (2) patients with coronary atherosclerotic heart disease that required coronary artery bypass surgery; and (3) patients with severe heart, liver, and kidney dysfunction who could not tolerate surgery.

\section{Data collection and follow-up}

All preoperative data and in-hospital outcomes were collected from our medical records database. Our database is an in-house computer system where patient variables, interventions, and outcomes are entered by active clinicians. TTE was performed preoperatively (G1) and 1 week (G2) and 3 months (G3) postoperatively for each patient. The left atrial anteroposterior diameter (LAD), left ventricular anteroposterior diameter (LVD), mitral orifice velocity (MV), mitral pressure of gradient (MVG), and ejection fraction (EF) were recorded and used to observe and compare the left heart reconstruction and mitral valve repair. 3D-TEE was performed before and during surgery to determine the degree of mitral valve insufficiency in real time.

\section{Apparatus and method}

Ultrasound was performed with a Philips EPIQ 7C with a S5-1 transthoracic probe and an $\mathrm{X7-2t}$ transoesophageal probe (Philips Ultrasound, Inc., Netherlands). 3D-TEE was used to select two atrial sections of the middle oesophagus to show the superior vena cava and inferior vena cava to prepare for femoral vein intubation under cardiopulmonary bypass. The Xplane program was then selected to concurrently display the biplane image of the leaflet to observe the opening and closing activity of the leaflet. The colour Doppler scale was adjusted to display the regurgitation of the valve leaflets, measure the regurgitation area, and evaluate the regurgitation degree. The 3D images of the mitral valve leaflets were obtained by 3D-ROOM, and their diagnosis and classification were performed according to the location of the mitral valve lesions and reverse flow. According to the Carpentier criteria, the mitral valve is divided into 8 regions: A1 (lateral anterior lobe), A2 (middle anterior lobe), A3 (medial anterior lobe), P1 (lateral posterior lobe), P2 (middle posterior lobe), P3 (medial posterior lobe), C1 (anterior lateral lobe junction), and C2 (medial posterior lobe junction). Before the operation, the sonographer carefully judged which area had the lesion, and MVQ software measured the left and right diameters of the annulus (DAIPm) to provide an imaging basis for clinicians to choose the appropriate valve model.

\section{Statistics}

Quantitative continuous variables are described as the means \pm standard deviation. Statistical differences between two or multiple groups were analysed by the SNK-q test or one-way analysis of 
variance with SPSS 23.0 software (IBM, Armonk, NY, USA). P $<0.05$ was considered statistically significant. The relationship between the valve replacement model and the DAIPm of the mitral valve annulus was analysed by logistic regression.

\section{Results}

Of the 58 patients enrolled, all were successfully intubated from the femoral vein to the upper and lower chambers of the right atrium under the guidance of 3D-TEE.

There were 40 men and 18 women (Fig. 1a) and the median age was 54 years. Baseline patient characteristics are reported in Table 1. Fifty-seven patients (98.28\%) underwent MVP under the 3D-TEE guided measurement of mitral valve structure-related indicators, and one patient underwent MVR due to the preoperative findings by 3D-TEE showing valvular stenosis. Analysis of the aetiology of the 57 cases of severe mitral valve regurgitation found the following: 3 cases of annulus enlargement, 2 cases of chordae tendineae rupture, 3 cases of valve leaflet perforation, and 49 cases of simple prolapse (Fig. 1b). The success rate of complete thoracoscopic MVP guided by real-time 3D-TEE was $100 \%$. There was no death or systolic anterior motion caused by mitral valve formation during the perioperative period. All patients were in a stable condition three months post operation. Three months after the operation, 3DTTE was performed again; the maximum forward flow velocity of the mitral valve orifice was $2.2 \mathrm{~m} / \mathrm{s}$, and the peak pressure difference was $19.36 \mathrm{mmHg}$. 
Table 1

Patients' baseline characteristics.

\begin{tabular}{|c|c|}
\hline & $N=58$ \\
\hline Age, yr & $54 \pm 16$ \\
\hline Female sex & $18(31)$ \\
\hline NYHA II & $21(36)$ \\
\hline NYHA III & $14(24)$ \\
\hline NYHA IV & $2(3)$ \\
\hline Acute left heart failure & $1(2)$ \\
\hline AF & $8(14)$ \\
\hline ASD & $2(3)$ \\
\hline Diabetes & $2(3)$ \\
\hline Hypertension & $17(29)$ \\
\hline CAD & $4(7)$ \\
\hline PDA & $1(2)$ \\
\hline pulmonary hypertension & $4(7)$ \\
\hline hyperthyroidism & $1(2)$ \\
\hline VTE & $7(12)$ \\
\hline 0 & $15(26)$ \\
\hline 1 & $17(29)$ \\
\hline 2 & $13(22)$ \\
\hline 3 & $2(3)$ \\
\hline 4 & $2(3)$ \\
\hline 5 & $2(3)$ \\
\hline \multicolumn{2}{|l|}{6} \\
\hline \multicolumn{2}{|c|}{ Values are presented as mean \pm standard deviation or $n(\%)$} \\
\hline \multicolumn{2}{|c|}{$\begin{array}{l}\text { Abbreviations: NYHA, New York Heart Association; AF: Atrial fibrillation; ASD: Atrial septal defect; CAD } \\
\text { coronary atherosclerotic heart disease; PDA: patent ductus arteriosus }\end{array}$} \\
\hline
\end{tabular}




\section{Comparison of the cardiac structure measurement indicators}

The results of the three ultrasound examinations showed statistically significant differences in EF, LAD and LVD (Table 1). EF in the first review after surgery was lower than that before surgery, which may be due to a temporary decrease in cardiac systolic function due to surgical trauma. In addition, EF was easily overestimated when there was moderate or worse MR before surgery. Three months after surgery, the heart structure returned to normal. While EF also tended to return to normal, it was still lower than the EF before surgery $(P<0.001$, Fig. $2 C)$. LAD and LVD were highest before surgery and decreased during recovery after surgery $(P<0.001$, Fig. $2 A, 2 B)$. The specific data are shown in Table 2.

Table 2

Left heart measurements

\begin{tabular}{|llllll|}
\hline Group & EF & LAD & LVD & MV & MVG \\
\hline G1 & $64.09 \pm 5.302^{\star \star}$ & $43.95 \pm 8.48^{\star \star}$ & $55.16 \pm 6.75^{\star \star}$ & $1.22 \pm 0.37$ & $6.50 \pm 3.79$ \\
G2 & $48.71 \pm 5.740$ & $40.05 \pm 7.71$ & $48.71 \pm 5.74$ & $1.19 \pm 0.32$ & $6.09 \pm 3.33$ \\
G3 & $61.78 \pm 9.584$ & $38.14 \pm 6.60$ & $47.69 \pm 4.90$ & $1.19 \pm 0.35$ & $6.11 \pm 3.85$ \\
\hline **P: Differences between groups; & & & \\
\hline
\end{tabular}

Comparison of the mitral regurgitation area

The preoperative transthoracic ultrasound measurement of the MR area (S1) and the intraoperative transoesophageal ultrasound measurement of the MR area (S2) was compared. S2 (9.57 \pm 1.47$)$ was greater than $S 1(8.83 \pm 2.01)$, and the difference between the two was statistically significant $(P<0.001$, Fig. 2F).

\section{The correlation between the ultrasonographic diagnosis of mitral valve prolapse and intraoperative diagnosis}

Intraoperative thoracoscopic diagnosis was used as the gold standard to judge the accuracy of TTE and 3D-TEE in diagnosing valve leaflet prolapse $(r=0.945,0.974)$. The accuracy of 3D-TEE was greater than that of TTE, and the difference between the two was statistically significant $(p<0.05)$. 3D-TEE accurately provides information on the anatomical structure and function of the mitral valve annulus, leaflets, chordae, papillary muscles, and atrioventricular walls to allow surgeons to better evaluate MR and improve its prognosis.

\section{Relationship between the DAIPm and the type of mitral annulus}

Logistic regression analysis was used to compare the relationship between DAIPm and the type of mitral annulus. The overall regression model was significant $(P<0.01)$. After adjustment, the R-square was 0.88 . 
The fitting effect of the two was good, indicating that DAIPm had significant predictive accuracy for the type of mitral annulus ( $p<0.05$, Fig. $4 A$ ). Among the 57 surgical patients, valve ring model $\# 30$ was the most frequently selected, followed by \#28 and \#32; \#34 was the least frequently used. The usage rate is shown in Fig. 4B.

\section{Discussion}

Primary MR is mainly caused by severe infective endocarditis, rheumatic heart disease, connective tissue disease, ischemic disease, and other diseases [9]. Regardless of the cause, MR can cause abnormal anatomical structure or function of the annulus valve, leaf valve, chordae tendineae, papillary muscle, and atrioventricular wall, which can aggravate regurgitation $[10,11]$. The most important treatments for MR are MVP and MVR. Almost all patients with MVR need to take anticoagulant drugs throughout their lives, and these patients have a relatively poor quality of life and a high possibility of other complications arising [12].

Thoracoscopic valvuloplasty requires only three holes to be made in the intercostal space of the right thoracic cavity; each hole is approximately $2-3 \mathrm{~cm}$ in diameter, and no median thoracotomy is required. The trauma to the patient is significantly reduced, allowing for quick patient recovery and short hospital stays. The patient with the shortest time in the ICU was 7 hours. Currently, MVP is the preferred treatment for MR, except in patients with ischemic lesions. MVP better preserves the left ventricular structure and functional status, reduces the occurrence of complications such as endocarditis, thromboembolism, and anticoagulation-related bleeding, and improves the long-term survival rate of patients. MVP involves a minimally invasive small incision, limited intraoperative field of vision, and limited operating space; therefore, the accurate preoperative diagnosis and accurate intraoperative positioning of the oesophageal ultrasound are essential.

\section{The measured value of TTE can be used as an objective indicator of the patient's surgical efficacy.}

In this study, transthoracic echocardiography was performed before the operation, 1 week after the operation, and 3 months after the operation. The relevant indexes of the cardiac structure measurements were then analysed. There were statistically significant differences in LAD, LVD, and EF, while there were no statistically significant differences in MV and MVG. After MVP, mitral valve regurgitation and volume load decreased significantly, the heart structure returned to normal, and LAD and LVD decreased. EF swiftly decreased in one week due to heart surgery trauma before returning back to normal. In a small number of patients, the mitral valve orifice flow rate increased, which may be due to the relative reduction of the valve orifice area by the sutures. In these patients, the valve orifice flow rate and pressure difference increased, but there was no statistical significance. Moreover, in the preoperative evaluation, the valve regurgitation area of 3D-TEE was more comprehensive than that of TTE. 3D-TEE was closer to the degree of intraoperative valvular insufficiency and could be evaluated objectively and accurately.

The location of the valvular disease and the choice of the forming ring are critical to the success of the surgery. 
Previously, the size of the ring was selected by using a petal meter. After forming the valve, a water injection test was performed in the left ventricle to observe whether lobular prolapse and regurgitation was still present. However, these operations were all observed in the empty state of the heart under cardiopulmonary bypass, which is very different from the physiological state of the normal filling and pulsation of the heart. Therefore, the positioning of the leaflet lesions and the selection of the valve annulus by these previous methods are of low reliability. Choosing an appropriately sized ring can minimize the mitral valve annulus, increase the effective area of the anterior and posterior valves, correct the mitral incomplete state, and reduce MR. 3D TEE can help surgeons accurately select the appropriate forming ring before surgery (Fig. 5).

In this study, it was found that the most frequently selected ring was \#30, followed by \#28. If the selected valve ring is too large, it cannot effectively fill the role of a contracture valve ring. If the selected base is too small, the base of the ventricular septum will be too thick, which may cause secondary left ventricular outflow tract stenosis. Therefore, it is vital during MVP to accurately locate the lobe lesion and select the appropriate size of the valve annulus. The relevant data showed that there were more prolapses in areas $\mathrm{A} 2$ and $\mathrm{P} 2$, and the correlation between the 3D-TEE diagnosis and intraoperative diagnosis of mitral valve prolapse was 0.958 , which indicated a good correlation. 3D-TEE, with its unique real-time threedimensional reconstruction capability and high resolution, was helpful for comprehensively analysing the condition of the MR valves, conducting accurate assessments, and providing reliable imaging for the selection of effective rings. In conclusion, 3D-TEE can be used to determine the aetiology and diagnosis of $\mathrm{MV}$, and it can be used to help reconstruct 3D images of the lobe lesions.

\section{The advantages of thoracoscopic surgery and the important role of "one-stop" echocardiography.}

The median sternotomy is a classical approach in cardiac surgery and allows enough exposure of the surgical field. However, the chest wall and sternum are severely injured by this operation; the patient endures a great deal of pain and wounds heal slowly and are prone to infection. In addition, this operation leaves a visible scar up to about $25 \mathrm{~cm}$ in the middle of the chest [13]. It had been reported that the minimally invasive MV surgery is popular due to its advantage over the robotic and standard approach [14].

When compared to traditional thoracotomy, the main advantages of minimally invasive thoracoscopic heart surgery are as follows: it avoids a large chest incision and sternotomy wound, significantly reduces tissue damage, and maintains the overall structure of the thorax [13]. Moreover, there is less bleeding in the operation field during and after the operation, and the pain is light, which can reduce the incidence of blood transfusion [15]. Patients can get out of bed in the early postoperative period, which is conducive to the postoperative recovery of elderly patients. Studies have shown that thoracoscopic MV surgery results in relatively less inflammation and injury reaction than median sternotomy [16]. This surgical method shortens the patient's stay in the intensive care unit and the number of days of hospitalization, reduces medical expenses, and relieves the psychological stress of patients in hospital. 
Since the skin incision is small and located on the side chest wall, the postoperative scar is relatively small and hidden, which better meets the patient's aesthetic needs.

Overall, TTE can provide an objective impression from the heart structure and function. Moreover, TTE is easy to operate, has good repeatability, and high acceptance among patients. It is the first choice for postoperative follow-up and condition monitoring.

3D-TEE can guide thoracoscopic extracorporeal circulation venous intubation, accurately locate valve leaflets, and reconstruct three-dimensional images of valve leaflets. It provides realistic imaging during examination that can assist cardiologists in formulating surgical plans, guide clinicians to quickly and accurately position the lesion site, evaluate immediate postoperative efficacy, and shorten the operation time. These advantages make it helpful in guiding the patient's postoperative recovery, providing the appropriate medication and nursing, and improving the patient's perioperative survival rate and long-term prognosis.

\section{Conclusion}

Perioperative echocardiography provides a one-stop diagnosis and treatment system for MR surgery. It can assist in preoperative diagnosis, intraoperative monitoring, and postoperative review, and ensure high-quality results. The combination of TTE and 3D-TEE is conducive to the treatment and rehabilitation of patients undergoing thoracoscopic MVP and can improve the quality of surgery and prognosis of patients. The combined application can also provide crucial guidance for clinical surgical operations, effectively improve the surgical outcome of patients, and reduce the incidence of complications.

\section{Abbreviations}

LAD

left atrial anteroposterior diameter

LVD

left ventricular anteroposterior diameter

MV

mitral orifice velocity

MVG

mitral pressure of gradient

$\mathrm{EF}$

ejection fraction

MR

mitral regurgitation

MVR

mitral valve replacement

MVP 
mitral valvuloplasty

TTE

Transthoracic echocardiography

TEE

Transoesophageal echocardiography

MVG

mitral pressure of gradient

$\mathrm{EF}$

ejection fraction

MR

mitral regurgitation

MVR

mitral valve replacement

MVP

mitral valvuloplasty

CDFI

Color Doppler flow imaging

DAIPm

the left and right diameters of the annulus

SAM

systolic anterior motion

\section{Declarations}

Ethics approval and consent to participate

The experiment has passed the ethical review of the hospital.

Consent for publication

All authors agree to publish.

Availability of data and materials

All data relevant to the study are included in the article.

\section{Competing interests}

The authors declare that there are no conflicts of interest.

\section{Funding}


This research was awarded the Zhejiang Provincial Health Science and Technology Project Plan (2021KY062).

\section{Authors' Contributions}

Ceng Wang was in charge of coordination, review, and correction of the manuscript.Cui Xiong was in charge for data analysis, draft and revise the manuscript. Other authors contributed to the conception and design of this work, its and interpretation, and all the authors are accountable for the integrity of this work. All authors read and approved the final manuscript.

\section{Acknowledgements}

Not applicable.

\section{References}

1. Asgar A, Ducharme A, Pellerin M, Garceau P, Basmadjian A, Bouchard D et al (2020) The Evolution of Transcatheter Therapies for Mitral Valve Disease: From Mitral Valvuloplasty to Transcatheter Mitral Valve Replacement. The Canadian journal of cardiology

2. Geyer M, Sotiriou E, Keller K, Tamm A, Ruf T, Kreidel F et al (2020) Feasibility of a MPR-based 3DTEE guidance protocol for transcatheter direct mitral valve annuloplasty. 37:1436-1442 Echocardiography (Mount Kisco, N.Y.). 9

3. Dreyfus G, Dulguerov F, Marcacci C, Haley S, Gkouma A, Dommerc C et al (2018) Respect when you can, resect when you should": A realistic approach to posterior leaflet mitral valve repair. J Thorac Cardiovasc Surg 156(5):1856-1866e3

4. Baumgartner H, Falk V, Bax J, De Bonis M, Hamm C, Holm P et al (2017) 2017 ESC/EACTS Guidelines for the management of valvular heart disease. Eur Heart J 38(36):2739-2791

5. Remenyi B, Webb R, Gentles T, Russell P, Finucane K, Lee M et al (2013) Improved long-term survival for rheumatic mitral valve repair compared to replacement in the young. World J Pediatr congenital heart Surg 4(2):155-164

6. Jiang Y, Wang C, Li G, Chen S (2021) Clinical outcomes following surgical mitral valve repair or replacement in patients with rheumatic heart disease: a meta-analysis. Annals of translational medicine 9(3):204

7. Gripari P, Mapelli M, Bellacosa I, Piazzese C, Milo M, Fusini L et al (2018) Transthoracic echocardiography in patients undergoing mitral valve repair: comparison of new transthoracic 3D techniques to 2D transoesophageal echocardiography in the localization of mitral valve prolapse. Int J Cardiovasc Imaging 34(7):1099-1107

8. Fatima H, Sharkey A, Qureshi N, Mahmood F, Mufarrih S, Baribeau V et al (2021) Three-Dimensional Transesophageal Echocardiography Simulator: New Learning Tool for Advanced Imaging Techniques. Journal of cardiothoracic and vascular anesthesia 
9. Chan V, Chu M, Leong-Poi H, Latter D, Hall J, Thorpe K et al (2017) Randomised trial of mitral valve repair with leaflet resection versus leaflet preservation on functional mitral stenosis (The CAMRA CardioLink-2 Trial). BMJ open 7(5):e015032

10. Kawamoto N, Fujita T, Fukushima S, Shimahara Y, Kume Y, Matsumoto Y et al (2018) Functional mitral stenosis after mitral valve repair for Type II dysfunction: determinants and impacts on longterm outcome. Eur $\mathrm{J}$ cardio-thoracic surgery: official J Eur Association Cardio-thoracic Surg 54(3):453-459

11. Madesis A, Tsakiridis K, Zarogoulidis P, Katsikogiannis N, Machairiotis N, Kougioumtzi I et al (2014) Review of mitral valve insufficiency: repair or replacement. J Thorac disease 6:S39-51

12. Kim J, Lee S, Joo H, Youn Y, Yoo K, Chang B et al (2021) Long-term Clinical Impacts of Functional Mitral Stenosis After Mitral Valve Repair, vol 111. The Annals of thoracic surgery, pp 1207-1215. 4

13. Yanagisawa J, Maekawa A, Sawaki S, Tokoro M, Ozeki T, Orii M et al (2019) Three-port totally endoscopic repair vs conventional median sternotomy for atrial septal defect. Surg Today 49(2):118-123

14. Hawkins R, Mehaffey J, Mullen M, Nifong W, Chitwood W, Katz M et al (2018) A propensity matched analysis of robotic, minimally invasive, and conventional mitral valve surgery. Heart 104(23):19701975

15. Deng L, Zhang G, Liu Z, Meng W, Liu H (2017) Totally thoracoscopic surgery for atrial myxomas resection and atrial septal defect repair. Eur Rev Med Pharmacol Sci 21(3):569-575

16. Jiang Q, Wang Z, Guo J, Yu T, Zhang X, Hu S (2021) Retrospective Comparison of Endoscopic Versus Open Procedure for Mitral Valve Disease. J Invest surgery: official J Acad Surg Res 34(9):1000-1006

\section{Figures}

GENDER

Man a Female

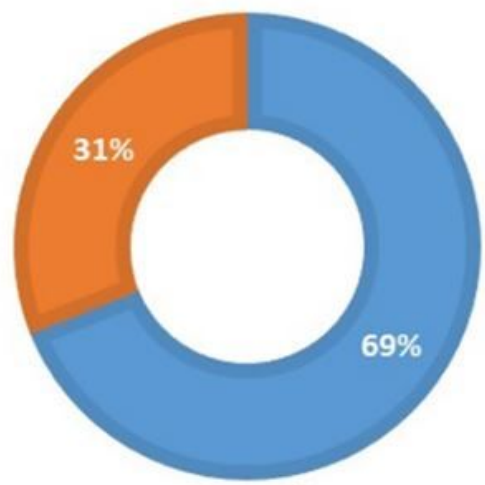

THE ETIOLOGY OF MR

- enlargement $\|$ chordae rupture $\square$ perforation $\|$ prolapse

B

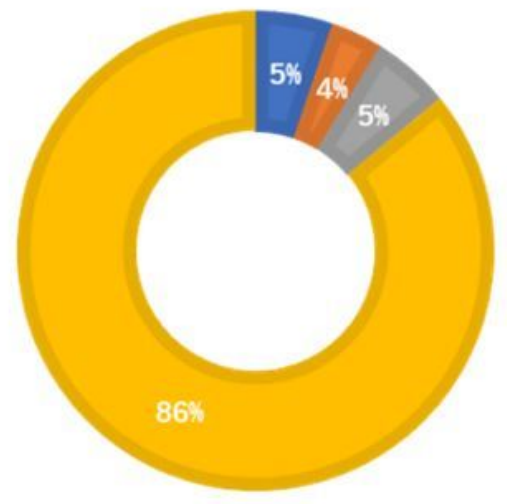


Figure 1

Figure A shows the ratio of men to women. Figure B shows the proportions of the various causes of mitral regurgitation.
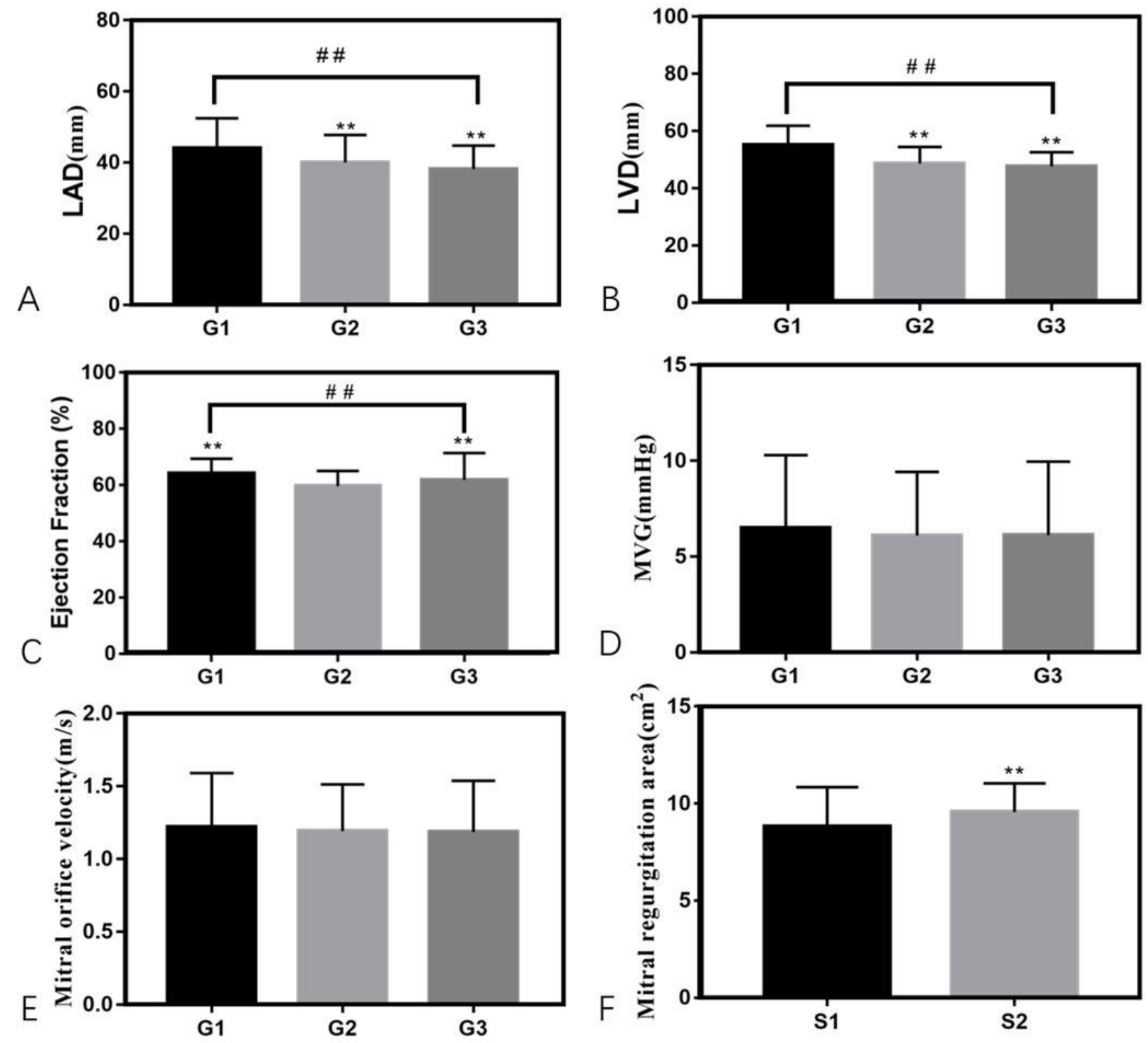

Figure 2

(A-E) Comparison of cardiac structure measurements by echocardiography.

(F) The area of mitral regurgitation was measured preoperatively by transthoracic ultrasound and intraoperatively by transoesophageal ultrasound. $* * \mathrm{P} \& \# \# \mathrm{P}<0.01$. 

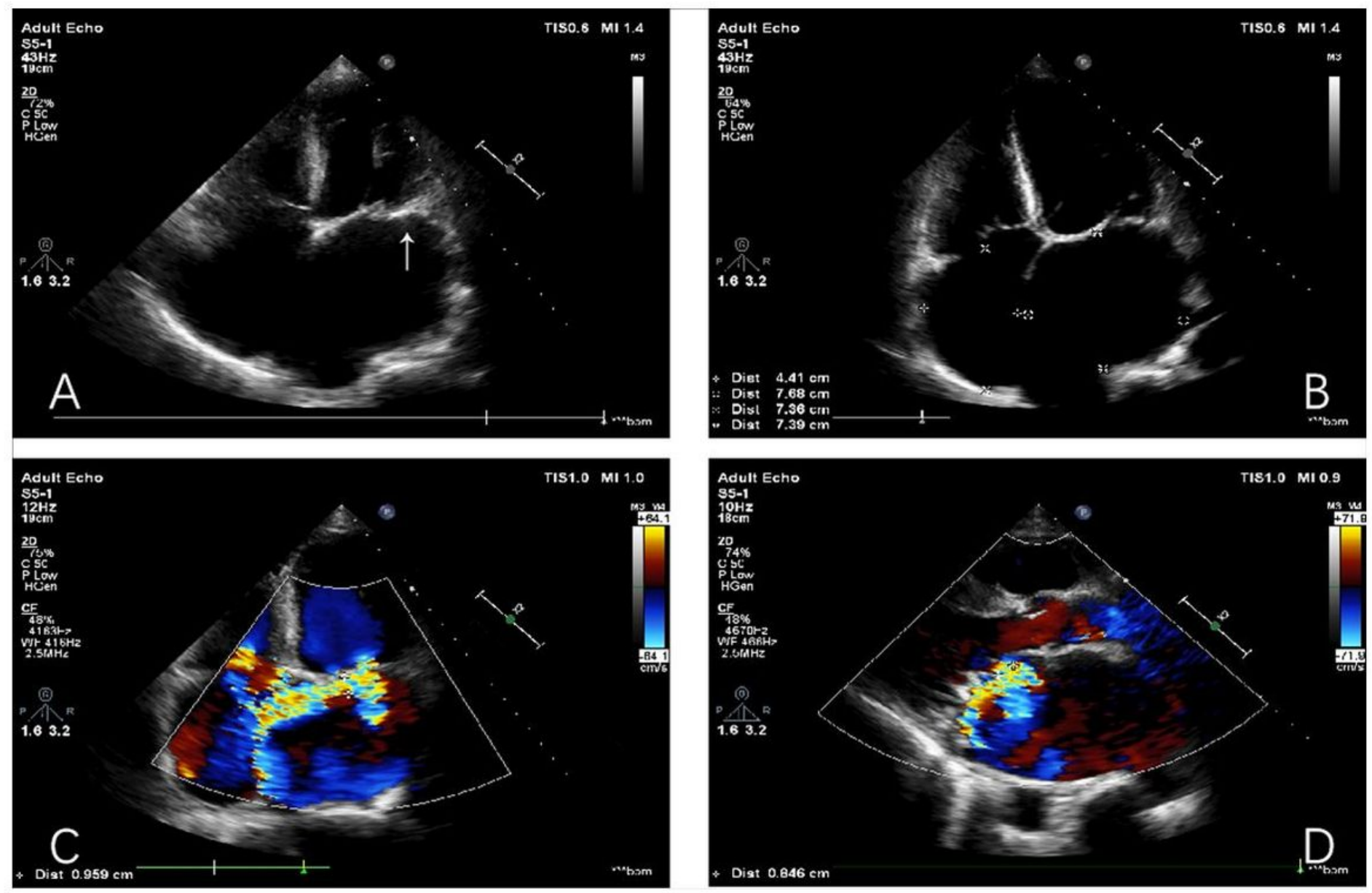

\section{Figure 3}

Transthoracic echocardiography. (A) Prolapse of the posterior mitral valve leaflet; (B) Mitral regurgitation causes enlargement of the left atrium; (C) Color Doppler flow imaging (CDFI) of the four-chamber view showing eccentric mitral regurgitation; (D) CDFI of the left ventricular long axis view showing eccentric mitral regurgitation. 


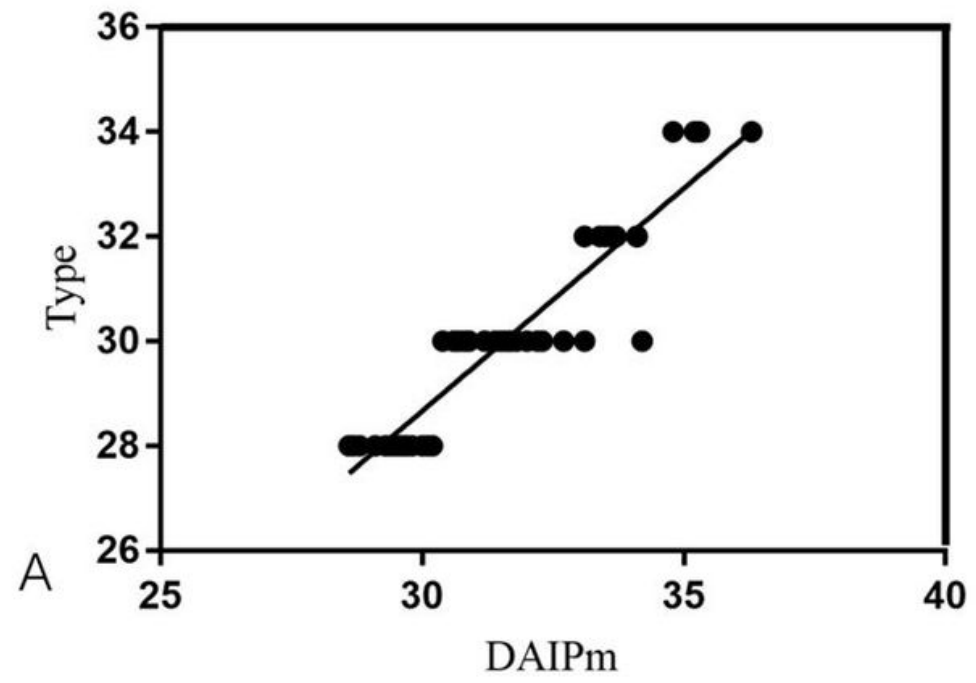

VALVE RING MODEL

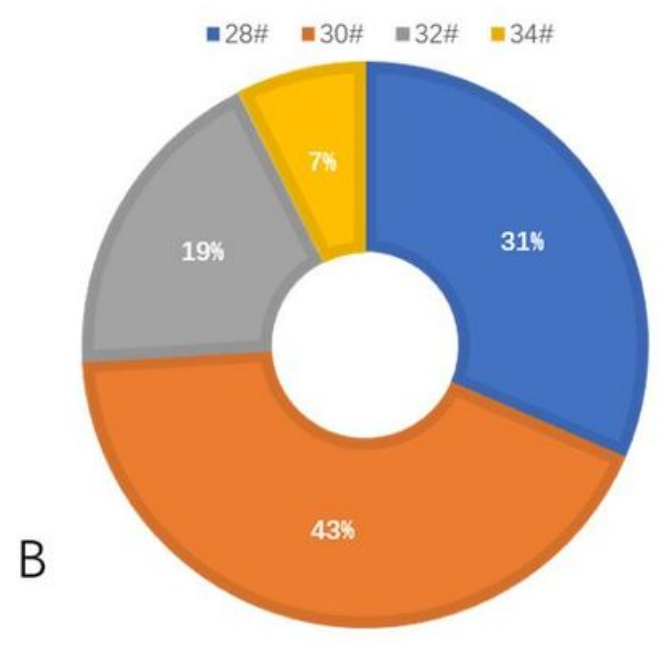

Figure 4

(A) Relationship between diameters of the annulus (DAIPm) and the type of mitral annulus. (B) Frequency of use of each model. 

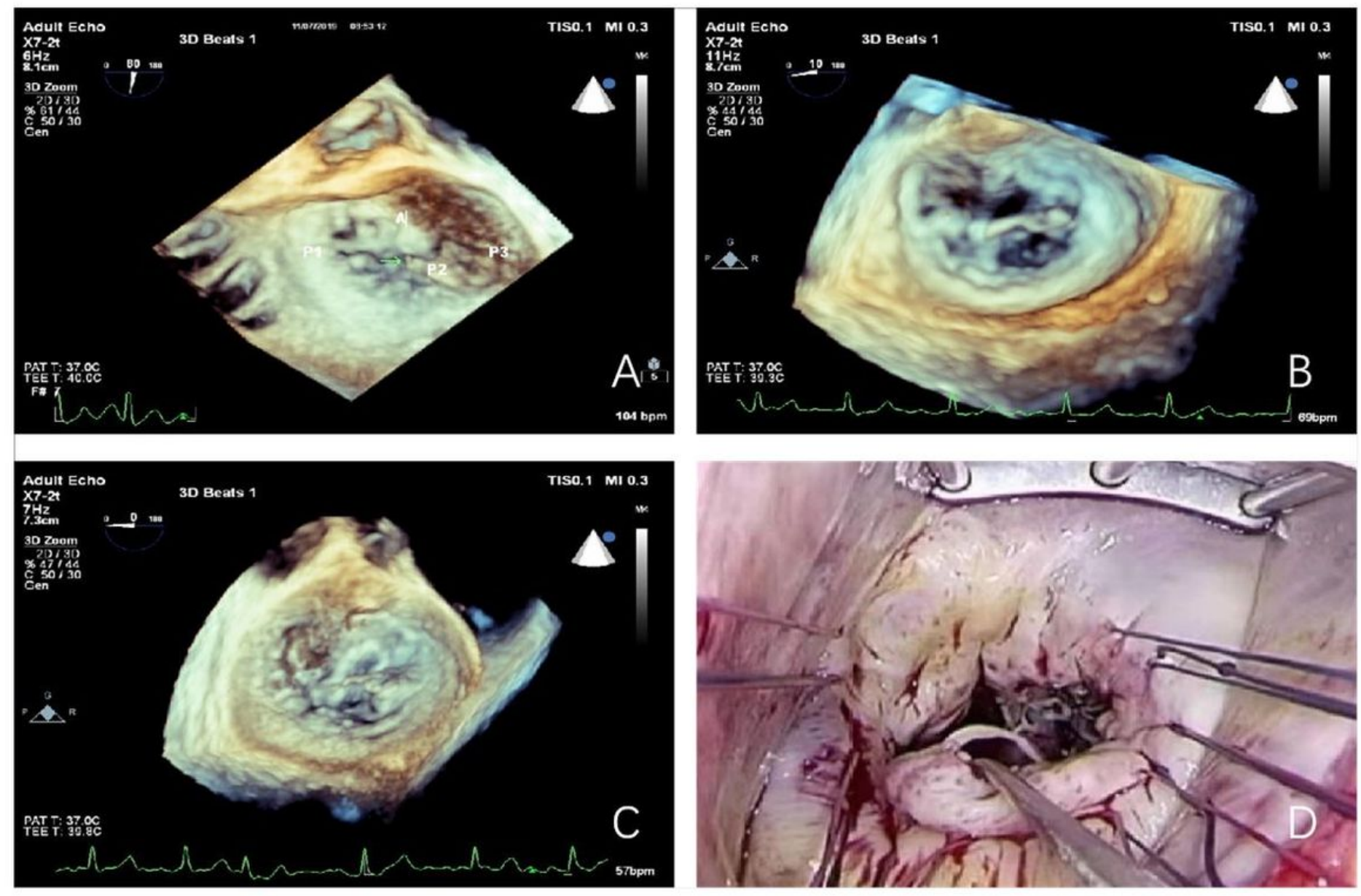

Figure 5

(A-C) Three-dimensional transoesophageal echocardiography imaging of the mitral valve. (D) Mitral valve under the field of thoracoscopic surgery. 